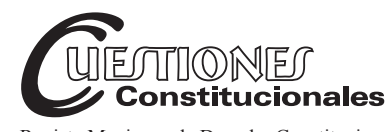

Revista Mexicana de Derecho Constitucional Núm. 42, enero-junio 2020

\title{
Regulación legal de las encuestas electorales: análisis teórico y comparado
}

\section{Legal Regulation of Election Polls: Theoretical and Comparative Analysis}

\author{
Pablo MARSHALL* \\ John CHARNEY** \\ Nelson RosAS***
}

RESUMEN: La importancia general que las encuestas electorales han adquirido y el impacto particular que se ha identificado que tienen en el periodo preelectoral han llevado a su regulación jurídica. Ello ha generado una discusión en torno a cuál es la regulación más adecuada para considerar los diferentes intereses en juego. El presente trabajo busca analizar críticamente la actual forma de regulación en el derecho comparado que, por un lado, establece un periodo blackout para la publicación de encuestas y, por otro, establece la obligación de disclosure para la comprensión y evaluación de los resultados de las encuestas. Se revisa el derecho comparado de forma panorámica y se ahonda en los casos de Estados Unidos, Canadá, España, Francia, Colombia y Chile. Se observa que las obligaciones de disclosure son menos problemáticas que el
ABSTRACT: The general importance and impact of election polls, particularly in the preelectoral period, have led to their legal regulation. This process has generated a complex discussion about the appropriate regulation to give room to the different interests at stake. This paper critically analyzes the current regulations in comparative law that, on the one hand, set a period of blackout for the publication of polls and, on the other hand, set an obligation of disclosure to provide information for the understanding and evaluation of the polls' results. Comparative law is presented in a panoramic way and in deep cases are studied: United States, Canada, Spain, France, Colombia and Chile. It is observed that the obligations of disclosure are less problematic than the

* Profesor de Teoría Constitucional, Facultad de Ciencias Jurídicas y Sociales de la Universidad Austral de Chile, Chile. Investigador DesDeh-UACh. Doctor por la Universidad de Glasgow. ORCID: 0000-0001-8347-4620; correo: pmarshall@uach.cl.

** Profesor e investigador del Centro Derecho Filosofía y Política, Facultad de Derecho de la Pontificia Universidad Católica de Valparaíso, Chile. Doctor por la Universidad de Londres (King's College). ORCID: 0000-0002-1971-1334; correo: john.charney@pucv.cl.ci.

***Egresado de la Facultad de Derecho de la Pontificia Universidad Católica de Valparaíso. ORCID: 0000-0001-8019-3479; correo: nelson.rosas.a@mail.pucv.cl. 
blackout en la medida que afecta de manera más tenue a las libertades de expresión e información. Sin embargo, al introducir algunos elementos teóricos que podrían servir para calificar y comprender mejor los intereses en juego, a saber, la libertad de expresión y el correcto funcionamiento de la democracia, se identifica una justificación robusta y satisfactoria no sólo para el disclosure sino también para el blackout.

Palabras clave: encuestas, elecciones, democracia, deliberación, libertad de expresión. blackouts because they seem to minimize the restriction of free speech and freedom of information. However, by introducing some theoretical elements that serve to qualify and understand correctly the collective democratic interests at stake, a robust and satisfactory justification is identified not only for disclosures but also for blackouts.

Keywords: Polls, Elections, Democracy, Deliberation, Free Speech.

\begin{abstract}
SUMARIO: I. Introducción. II. Un marco conceptual para la regulación de las encuestas. III. Derecho comparado. IV. La regulación desde una perspectiva teórica: el argumento deliberativo. V. Conclusiones. VI. Bibliografía.
\end{abstract}

\title{
I. INTRODUCCIÓN
}

Las encuestas electorales forman parte del panorama de la política contemporánea. Ellas pueden cumplir varios roles importantes en los sistemas políticos democráticos. Pueden ser un instrumento que permite a quienes detentan posiciones de poder medir determinadas preferencias de la ciudadanía y adoptar decisiones de acuerdo con ellas. Permiten, además, a quienes están disputando el poder político, durante periodos de elecciones, tomar decisiones estratégicas para captar el voto de uno u otro sector de la ciudadanía. Ellas también pueden proporcionar información a la ciudadanía sobre las preferencias electorales en un momento determinado. Las encuestas, finalmente, pueden servir como una forma de control democrático allí donde los gobiernos se sienten tentados a manipular fraudulentamente las elecciones.

La necesidad de regulación de las encuestas ha sido la consecuencia de un conjunto de problemas que ellas implicarían para el normal funcionamiento del sistema democrático, en especial el de conducir las decisiones electorales a cuestiones muchas veces desvinculadas de asuntos políticos de carácter sustantivo. La discusión constitucional en torno a su regulación se ha centrado en su potencial afectación de ciertas libertades, como la libertad de ejercer una actividad económica lícita, la libertad de la prensa de publicar la información levantada y la libertad de los ciudadanos de 
recibir dicha información. Quienes critican la regulación de las encuestas electorales no sólo apelan a la importancia, cantidad e intensidad de libertades afectadas, sino que también aseguran la no existencia de evidencia que demuestre que ellas producen efectos perjudiciales para el sistema democrático. Por otro lado, quienes promueven su regulación apelan a la importancia que la discusión sobre asuntos políticos sustantivos ocupa en un sistema democrático y a la necesidad de proteger y mejorar la deliberación democrática acerca de esos asuntos de los efectos potencialmente corrosivos de las encuestas electorales, en especial durante periodos de elección. La pregunta que subyace a este trabajo es, por tanto, si las razones existentes para regular legalmente las encuestas electorales pueden legítimamente limitar derechos fundamentales que podrían expresarse a través de su realización, publicación y difusión.

El principal objetivo de este trabajo es articular una defensa de la necesidad de regular las encuestas electorales en periodos de elecciones populares. Lo haremos sobre la base del argumento deliberativo. Nuestra hipótesis es que, si volcamos nuestra comprensión de la libertad hacia un concepto de libertad esencialmente político y sensible a las formas de dominación que las encuestas ejercen sobre la ciudadanía a través de la degradación de la discusión pública, la regulación de las encuestas electorales no sólo es permisible sino incluso necesaria, y que tiene como implicancia, en términos de los intereses en juego, que las libertades comunicativas involucradas deben ceder ante la protección de una esfera pública democrática. Para confirmar dicha hipótesis, el desarrollo de la argumentación será el siguiente. Primero, se realizan algunas precisiones conceptuales y distinciones que ayudarán a entender con mayor precisión el objeto de estudio, las alternativas regulatorias disponibles y los problemas que éstas han enfrentado. Segundo, se revisa de forma sinóptica la forma en que el derecho comparado ha regulado la producción y difusión de encuestas electorales, al tiempo que se identificarán algunos casos que ejemplifican la forma como se ha llevado a cabo el control judicial de constitucionalidad de estas medidas. Tercero, se presentará un análisis de la teoría constitucional que identifique el problema subyacente a la restricción de la libertad para publicar encuestas electorales, que no ha sido correctamente retratado por el análisis de jurídico-constitucional. Abordando insumos provenientes de las tradiciones liberal y democrática, se ofrecerán razones que tienen relación con la concepción de las libertades 
involucradas, que permitirán avanzar en una justificación robusta, no sólo de la necesidad de limitar la opacidad de la información provista por las encuestas, sino también de limitar sus efectos.

\section{UN MARCO CONCEPTUAL PARA LA REGULACIÓN DE LAS ENCUESTAS}

Si se observa la literatura, se pueden sacar algunas conclusiones preliminares y trazarse algunas distinciones que pueden orientar una exposición más fecunda del análisis comparado y teórico que se realiza en las dos secciones siguientes.

\section{Tipos de encuestas}

Una primera cuestión que hay que aclarar es la variedad de fenómenos que muchas veces se asocian a la noción de encuestas electorales. Para el propósito de determinar el objeto de análisis de este artículo es necesario distinguir entre diferentes modos de investigación social vinculado a la política y las elecciones. En primer lugar, se debe distinguir claramente entre encuestas de opinión sobre asuntos de interés público y encuestas electorales o de intención de voto. Mientras las primeras indagan el apoyo a ciertas políticas o medidas, o el apoyo a ciertas autoridades, las segundas se enfocan en intentar recoger la intención de los votantes de cara a una elección venidera. Dicho de otro modo, las primeras son encuestas que buscan determinar la opinión actual de la ciudadanía sobre ciertas cuestiones sustantivas y las segundas son encuestas que buscan realizar una predicción del comportamiento electoral futuro de la ciudadanía. Por supuesto que las encuestas de opinión, cuando son realizadas en los días previos a la elección, y especialmente cuando la campaña electoral se ha centrado en ciertos asuntos claves, podría tener un carácter predictivo, pero éste sería, sin duda, mucho menos específico que el de una encuesta de intención de voto. ${ }^{1}$

En segundo lugar, se debe distinguir entre las encuestas preelectorales de intención de voto de todas aquellas operaciones que tienen como obje-

\footnotetext{
1 Donsbach, Wolfgang y Hartung, Uwe, "The legal Status of Public Opinion Research in the World", en Donsbach, Wolfgang y Traugott, Michael W. (eds.), The Sage Handbook of Public Opinion Research, Los Angeles, Sage Publications, 2008, p. 432.

Esta obra está bajo una Licencia Creative Commons

Atribución-NoComercial-SinDerivar 4.0 Internacional, IIJ-UNAM.
} 
tivo la proyección de resultados de las preferencias ya marcadas. Mientras las primeras intentan predecir un hecho futuro, las segundas intentan reconstruir, con base en una información incompleta, el resultado todavía no oficialmente anunciado de la elección. Entre este segundo tipo de método, destacan por su popularidad las encuestas de salida de la urna (exit polls) que preguntan a los votantes cuál ha sido su preferencia marcada, a las que se suman proyecciones matemáticas para anticipar un resultado. La popularidad de este tipo de instrumento está en franco declive, especialmente ante el perfeccionamiento técnico que hace que los resultados oficiales estén disponibles, en muchos países, sólo después de un par de horas del cierre de los locales de votación. ${ }^{2}$

Finalmente, debe distinguirse, por un lado, entre encuestas privadas, que sirven a las organizaciones que las practican para orientar su acción, como, por ejemplo, las encuestas privadas que realizan los partidos políticos para identificar la competitividad de sus candidatos y, por otro, las encuestas públicas, que son aquellas encuestas cuyos resultados son presentados a la opinión pública por su carácter noticioso o por su potencial para generar un efecto en el electorado. ${ }^{3}$

El análisis que sigue se enfoca en el caso de encuestas electorales previas a la elección y de carácter público.

\section{Efectos de las encuestas electorales}

La necesidad de restringir el mercado de las encuestas electorales se ha venido dando por la percepción de que ellas tienen algún efecto en el proceso electoral que puede ser evitado mediante una intervención regulatoria. La revisión de la literatura permite identificar dos tipos de efectos de las encuestas de intención de voto: primero, aquellos efectos que se producen en la dinámica política del proceso electoral en general (afectando a mediano y a largo plazo, tanto a los actores como al sistema político, a las instituciones políticas y al sistema de medios); y segundo, aquellos efectos

\section{Idem.}

3 López, Miguel Ángel et al., "Sobre experiencia internacional en regulación y autorregulación de encuestas políticas y electorales de opinión pública. Lecciones para Chile", en De la Fuente, Gloria (ed.), Calidad de la democracia, transparencia e instituciones políticas, vol. 1, Santiago de Chile, Segpres y Lom Ediciones, p. 225. 
en el comportamiento electoral individual de los ciudadanos (que afectan directamente - y a corto plazo - a los ciudadanos en cuanto electores). ${ }^{4}$

Respecto a sus efectos generales o macro-electorales, la preeminencia de las encuestas electorales sobre otro tipo de contenido informativo en el contexto de la campaña electoral, ha generado lo que ha sido denominado horse-race effect, que se refiere a un estilo de campaña electoral, que se asemeja a una carrera de caballos, en la medida que todo el foco de atención se dirige a la información de las encuestas que indican qué candidato está ganando o cuáles son los márgenes de predominio de un candidato por sobre la percepción del público, acerca de los programas políticos de los candidatos o de los asuntos de relevancia pública, que diferencian las políticas que los candidatos implementarían de resultar electos. Este efecto ha transformado de tal modo las campañas electorales, que en muchos casos las encuestas han desplazado a las elecciones primarias como mecanismo de selección de candidatos por parte de los partidos políticos.

Con relación a los efectos de las encuestas electorales en el comportamiento de los votantes o microelectorales, se han identificado varios efectos. Los más debatidos son el bandwagon effect o el efecto de convencer a los electores para votar por el candidato supuestamente ganador, y el underdog effect o el efecto de convencer a los electores para votar por el candidato que va perdiendo, pero también son mencionados el efecto motivador o desmotivador, que convence a los electores de ir o no a votar dependiendo de si su voto va a ser relevante en la disputa electoral, o el efecto de generar un voto estratégico, que sucede cuando un elector decide votar por un candidato que no representa su posición ideológica en pos de marcar una preferencia que pueda tener un impacto al resultado final de la elección. ${ }^{5}$ Mientras algunos electores toman en cuenta las encuestas a la hora de tomar decisiones, otros electores pueden ser afectados por dichos resultados sin estar conscientes de ello. ${ }^{6}$ El principal problema que este segundo tipo de efecto genera es que se plantean una serie de incentivos para los actores políticos en competencia, y para las fuerzas que los apoyan para manipular o simplemente crear resultados que produzcan el efec-

\footnotetext{
4 Donsbach, Wolfgang y Hartung, Uwe, op. cit., p. 433.

5 Sobre estos efectos, véase Dahlgaard, Jens Olav et al., "How are Voters Influenced by Opinion Polls? The Effect of Polls on Voting Behavior and Party Sympathy", World Political Science, Copenhagen, 12 (2), 2016, pp. 283-300.

6 Feasby, Colin C. J., "Public Opinion Poll Restrictions, Elections, and the Charter", $U$. Toronto Fac. L. Rev., Toronto, 55, 1997, p. 244. 
to deseado. ${ }^{7}$ Los principales detractores de la regulación de las encuestas electorales insisten en señalar, no obstante, la falta de evidencia en torno a estos efectos microelectorales. ${ }^{8}$

\section{Tipos de regulación legal de las encuestas}

Dejando de lado la autorregulación que las propias asociaciones de encuestadores establecen, muchas de las cuales pueden ser extraordinariamente exigentes, ${ }^{9}$ la regulación legal de las encuestas electorales se ha concentrado en el uso de dos mecanismos: el blackout o embargo, y el disclosure o ficha técnica.

El embargo o blackout consiste en prohibir la realización, publicación o retransmisión del resultado de las encuestas electorales durante un periodo variable previo a la elección. Este primer mecanismo es considerado una regulación más invasiva y restrictiva del derecho a la información.

El tiempo del embargo varía mucho, y esto se puede deber a una pluralidad de justificaciones que se ofrecen. Un embargo más corto puede obedecer a la necesidad de crear un periodo en el cual los candidatos puedan responder y criticar encuestas que han sido erróneamente comprendidas o deliberadamente manipuladas. Un embargo más largo, al final del periodo de campaña, puede estar diseñado para establecer un momento en que los electores puedan reflexionar en los méritos relativos de los candidatos y partidos, sin prestar atención a su rendimiento en las encuestas. Ambas funciones, sin embargo, pueden concurrir en un embargo que se extiende, por ejemplo, por las últimas dos semanas del periodo de campaña electoral. Por último, un embargo largo, que pudiera extenderse inclusive durante todo el periodo de la campaña electoral, podría servir para volver a enfocar la campaña y la actividad noticiosa que gira en torno a ella en asuntos políticos sustantivos, alejados del estilo deportivo generado por

\section{Ibidem, p. 248.}

8 Véase el caso de estudio de México en De la Peña Mena, Ricardo, "El debate sobre las encuestas electorales en México en 2012”, Revista Mexicana de Opinión Pública, México, vol. 20, enero-junio de 2016, pp. 53-80.

9 Véase, por ejemplo, Esomar y Wapor, ESOMAR/WAPOR Guideline on opinion polls and published surveys, Lincoln, Nebraska, 2014, disponible en: https://www.esomar.org/ uploads/public/knowledge-and-standards/codes-and-guidelines/ESOMAR-WAPOR-Guideline-on-Opinion-Polls-and-Published-Surveys-August-2014.pdf, consultado el 26 de noviembre de 2018. 
las encuestas electorales. Esto permitiría que la elección democrática informada y deliberada en torno a esos asuntos sustantivos sea posible de imaginar. ${ }^{10}$

Un segundo tipo de regulación consiste en la obligación de acompañar los resultados de las encuestas con una ficha técnica o disclosure, esto es, cierta información relevante que permite entender el valor y confiar en los resultados publicados. Esta es considerada una regulación menos intrusiva y más respetuosa de la libertad de expresión e información en la medida que no prohíbe, sino que sólo establece un requisito para la publicación.

Hay tres aspectos que buscan ser abordados por esta clase de medida. En primer lugar, las instituciones que se dedican a realizar y publicar encuestas electorales tienden a tener afiliaciones ideológicas. Ese es el caso evidente de las empresas con fines de lucro que se dedican al negocio de las encuestas, su afiliación ideológica es la de sus propietarios. Pero también, puede ser el caso de las universidades o los medios de comunicación. Que estas instituciones tengan una preferencia ideológica determinada nada dice de que ellas necesariamente vayan a presentar información sesgada o que intenten interpretar la información recopilada para favorecer sus propias inclinaciones electorales, pero sí transforma en cuestionable la fe ciega en el valor científico de dicha información que parece mostrarse en ciertas circunstancias. Esta preocupación hace relevante contar con información transparente respecto a quién realizó la encuesta, quién es el responsable de su publicación y quién ha financiado los costes de su realización. Ello permitirá leer la encuesta a través de un prisma menos.

En segundo lugar, debe atenderse al riesgo de manipulación de la información provista por las encuestas. Esto es posible cuando falta la información sobre las muestras. La poca atención que se presta al margen de error y la opacidad con relación a las preguntas puede hacer pensar que los datos aportados pueden ser manipulables sin mayores consecuencias. En este sentido, es fundamental que la ficha técnica incorpore información que permita un análisis metodológico de la información publicada, que incluya, entre otras cosas, el objetivo del estudio, la población estudiada, el marco muestral, la descripción del diseño de la muestra, el tamaño de la muestra, el fraseo exacto de las preguntas, los criterios de elegibilidad de los encuestados, el margen de error, el método de levantamiento, la tasa

\footnotetext{
10 Feasby, Colin C. J., op. cit., p. 251.
} 
de rechazo de la entrevista, las fechas de levantamiento, el procedimiento de estimación y la supervisión.

En tercer lugar, en la medida que la información de la ficha técnica es comúnmente comprensible, sólo por personas especialistas, hay una preocupación respecto de que cierta información general e importante sea puesta en conocimiento del público en un lenguaje que pueda ser comprendido por todos los ciudadanos, y con una explicación del significado de sus aspectos técnicos fundamentales, como el margen de error o las características básicas de la muestra. En la medida que dicha información tenga que ser presentada de manera explícita y clara, una obligación de contar con una nota explicativa podría disuadir la realización de encuestas de mala factura técnica, que se realizan con el puro ánimo de manipular a la opinión pública.

Como puede observarse, la obligación de publicación de la ficha técnica no supone una restricción a la publicación de encuestas, que no cumplen con estándares metodológicos mínimos o cuyo financiamiento evidencia un claro conflicto de interés. Por el contrario, la obligación de su publicación descansa en que la opinión pública tiene la capacidad de realizar un juicio sobre esa información. Quienes dudan de la capacidad de la ciudadanía de analizar críticamente las encuestas sirviéndose de la ficha técnica, han sugerido una forma de control aún más ambiciosa. Un tercer mecanismo regulatorio busca garantizar el uso obligatorio de estándares metodológicos adecuados por parte de las encuestas políticas. A ello, se puede llegar a través del mecanismo de la certificación de las encuestas por parte de un organismo experto y oficial, o por medio de la obligación de seguir ciertas normas oficiales que aseguren la objetividad y la calidad de la información hecha pública. Nada de ello puede funcionar sin la amenaza de sanciones por la publicación de resultados no certificados o que infrinjan las normas oficiales. Sin embargo, también pueden encontrarse mecanismos regulatorios que funcionan sobre la base de sanciones, allí donde la publicación de las encuestas se haya hecho alterando los resultados o mediante manipulaciones o deformaciones de estos últimos.

\section{Problemas de la regulación}

La primera y más importante de las críticas contra le regulación de las encuestas electorales se ha dirigido contra su carácter restrictivo de la li- 
bertad de expresión e información. ${ }^{11}$ Especialmente cierta, con relación a los embargos, la pregunta subsiguiente es si dicha restricción en las libertades que protegen la libre circulación de la información en la esfera pública democrática, puede estar justificada y si dichas justificaciones son suficientemente vigorosas para revertir la presunción en favor de la libertad de expresión e información a la que nos hemos acostumbrado. Esta crítica, cuya concreción institucional es el reclamo de la eventual inconstitucionalidad de una regulación demasiado intrusiva, invita a presentar los intereses en juego como intereses contrapuestos: por un lado, la libertad individual constitucionalmente protegida y, por otro, el interés público en limitar dicha libertad individual en busca de la satisfacción de un bien público.

Esto nos lleva al segundo orden de críticas que se han formulado a la regulación que sostiene que no puede haber una clara justificación donde no existen pruebas del efecto que la difusión del resultado de las encuestas puede producir en el electorado. Como se señalará, la literatura sobre este particular no ha llegado a un consenso sobre la existencia de efectos particulares o microelectorales en el comportamiento de los votantes. ${ }^{12}$ Sin embargo, debe recordarse que los efectos de la difusión de dichos resultados pueden no sólo ser a nivel individual, sino que también pueden afectar la dinámica general del proceso electoral (macroelectorales) en formas que podrían querer evitarse. ${ }^{13}$

Finalmente hay una serie de objeciones de carácter práctico. La determinación precisa del plazo del embargo ha sido criticada en la medida que la variabilidad de su duración en el derecho comparado no parece obedecer a una racionalidad determinada. ¿Cómo determinar racionalmente la duración del embargo? Asimismo, se han planteado dudas sobre la posibilidad de hacer cumplir el embargo en un entorno digital que no conoce de fronteras jurisdiccionales cuando de lo que se trata es de acceder a información. Pero no sólo el carácter transnacional de la web supone un problema, la posibilidad de la acelerada e ilimitada reproducción de contenido digital a través de redes sociales nos debe llevar a concluir que la

11 También se mencionan la libertad de investigación científica, la libertad de iniciativa económica y la libertad de prensa. Donsbach, Wolfgang, Who's Afraid of Election Polls? Normative and Empirical Arguments for the Freedom of Pre-election Surveys, Amsterdam, Foundation for Information, Esomar, 2001, pp. 7 y 8.

12 Idem.

13 Feasby, Colin C. J., op. cit., p. 245. 
restricción del flujo de información debe también dar cuenta, si es que eso es posible, de los cambios tecnológicos. ${ }^{14}$

\section{DERECHO COMPARADO}

\section{Una visión panorámica de la regulación comparada}

Como lo hemos adelantado, el análisis de la experiencia regulatoria en el derecho comparado deja al descubierto determinados patrones, los cuales permiten agrupar a los Estados en torno a dos tendencias que, aunque con particularidades que varían en cada país, son generalmente identificables. Así, los países anglosajones se caracterizan por recurrir a la técnica de la autorregulación, en el sentido que los lineamientos de ética y de calidad de la industria son provistos por la misma comunidad de encuestadores a los que van dirigidos. Este es el caso, por ejemplo, de la práctica de los Estados Unidos de América, Reino Unido y Australia. ${ }^{15}$

En la otra vereda, los países que han optado por el camino de la regulación legal para fijar estos criterios reciben en su mayoría inspiración de los principios del derecho continental. Aquí encontramos en la actualidad a gran parte de la comunidad internacional. Conforme al más reciente reporte llevado a cabo a nivel mundial, en 2018 un 60\% de los 133 países consultados contaba con la existencia de embargos o blackouts legales a la publicación de encuestas. ${ }^{16}$ Particularmente estricta es la regulación en ciertos países, en donde las encuestadoras se encuentran, en adición a los embargos, con obligaciones de publicación de fichas técnicas. Como lo demuestra además este estudio, es posible observar en los últimos años un aumento en el número de países que han optado por este último modo de abordar el problema. Tal es el caso de la mayor parte de los países de América Latina, en donde la existencia de embargos, por un número variable de días anteriores a las elecciones, es ahora un denominador común.

\footnotetext{
14 Donsbach, Wolfgang, op. cit., pp. 8 y 9.

15 López, Miguel Ángel et al., op. cit., p. 228

16 Esomar y Wapor, Freedom to Conduct Opinion Polls, A 2017 Worldwide Update, Lincoln, Nebraska, 2018, pp. 6 y ss., disponible en: https://wapor.org/publications/freedom-topublish-opinion-polls/, consultado el 27 de noviembre de 2018.
} 
Es difícil precisar cuál de estas dos orientaciones es la más efectiva, considerando que además tampoco hay claridad, como lo hemos señalado, respecto a los efectos que las encuestas tienen en el electorado. Con todo, parece al menos haber consenso sobre la deseabilidad de resguardar la calidad de sus resultados. En este sentido, ambas formas de regulación buscan asegurar, con mayor o menor intensidad y poniendo el foco de atención en distintos aspectos, la observación de unos estándares de calidad mínimos en la información, que es entregada finalmente al público, toda vez que existe la conciencia generalizada por parte de los reguladores de que el conocimiento adecuado de la opinión pública cumple un rol fundamental en las democracias modernas. El contraste de fondo, antes bien, debemos hallarlo en diferencias más profundas de tipo político que se expresan en el distinto valor que se le asigna en cada sistema normativo a las libertades de expresión e información constitucionalmente protegidas. Estas libertades, según quienes favorecen la autorregulación, no permitirían su restricción por parte del Estado en el caso de las encuestas electorales. ${ }^{17}$ Por el contrario, donde sí existen restricciones legales, éstas son usualmente justificadas teniendo a la vista una concepción normativa del sistema democrático, conforme a la cual es necesario restringir ciertas libertades en orden a procurar una democracia saludable. ${ }^{18}$ Forman parte de esta concepción la consecución de resultados electorales que reflejen de la mejor manera posible los genuinos intereses del electorado. Por supuesto, estas consideraciones no son ajenas para los países angloparlantes y de hecho no son inusuales los casos de encuestadores que han elegido no publicar sus hallazgos en los días previos a los comicios. ${ }^{19}$ Sin embargo, cuando estas restricciones han sido establecidas en los códigos de conducta voluntarios, su justificación suele apelar a la ética del encuestador, aunque también hay quienes han mencionado con este fin a la necesidad de resguardar "la dignidad del proceso democrático". ${ }^{20}$

17 Orr, Graeme y Levy, Ron, "Regulating Opinion Polling: A Deliberative Democratic Perspective", UNSW Law Journal, Sydney, 39 (1), 2016, p. 319.

18 Donsbach, Wolfgang y Hartung, Uwe, op. cit., pp. 435 y ss.

19 Petersen, Thomas, "Regulation of Opinion Polls: A Comparative Perspective", en HoltzBacha, Christina y Strömbäck, Jesper (eds.), Opinion Polls and the Media, Reflecting and Shaping Public Opinion, Nueva York, Palgrave Macmillan, 2012, p. 53.

20 Frankovic, Kathleen, "Exit Polls and Pre-Election Polls", en Donsbach, Wolfgang y Traugott, Michael W. (eds.), The Sage Handbook of Public Opinion Research, Los Angeles, Sage Publications, 2008, p. 578. 
En lo que resta de esta parte del trabajo, se revisan algunos ejemplos de regulación legal de encuestas electorales y de revisión de su constitucionalidad. La selección de los ejemplos es anecdótica, aunque hemos intentado retratar el panorama internacional identificando casos representativos de la regulación de los sistemas jurídicos del common law (Estados Unidos de América y Canadá), de los sistemas jurídicos continentales (España y Francia) y de los sistemas jurídicos latinoamericanos (Colombia y Chile) que, si bien han seguido el modelo continental, son de interés en cuanto experiencias regionales que han abordado el fenómeno. ${ }^{21}$

\section{Estados Unidos de América}

Siendo los Estados Unidos de América la cuna de la industria electoral es interesante revisar la discusión que allí ha tenido lugar. En efecto, las primeras encuestas practicadas en los Estados Unidos de América datan del siglo XIX ${ }^{22}$ y nacen de la mano con el periódico como medio pionero de la comunicación de masas. Si bien, ya en 1824, las encuestas fueron utilizadas por primera vez para predecir el resultado de las elecciones presidenciales, ${ }^{23}$ no fue sino hasta la década de los 1930 que su uso se convertiría en una herramienta importante para el proceso electoral. Su calidad por estos años era deficiente y sus resultados poco confiables. La necesidad de contar con buenas predicciones impulsó de esta manera el nacimiento de las encuestas cientificas, las que pese al éxito inicial experimentado en los comicios de 1936, y de haber ganado el interés nacional, pronto experimentarían un revés mayor, cuando en 1948 equivocadamente

21 Véase asimismo otros casos latinoamericanos: sobre México, Corzo Sosa, Edgar, "Las encuestas electorales y los derechos fundamentales: una primera reflexión", Cuestiones Constitucionales. Revista Mexicana de Derecho Constitucional, México, núm. 16, enero-junio de 2007, pp. 71-87, disponible en: https://revistas.juridicas.unam.mx/index.php/cuestiones-constitucionales/article/view/5788/7628, consultado el 21 de agosto de 2019; y Sánchez, Enrique Inti García, "Criterios legales y jurisdiccionales vinculados a encuestas electorales en México", Revista Mexicana de Estudios Electorales, México, núm. 16, 2016, pp. 126-138; sobre Argentina, véase Alonso, Belén, "Las encuestas en los medios. Un estudio sobre su regulación en Argentina desde el tecnicismo y la política", XXV International Congress Latin American Studies Association (LASA), Las Vegas, octubre de 2004.

22 Streb, Matthew J. y Genovese Michael A., "Polling and the Dilemmas of Democracy", en Streb, Matthew J. y Genovese, Michael A. (eds.), Polls and Politics, The Dilemmas of Democracy, Albany, State University of New York Press, 2004, pp. 9 y ss.

23 Frankovic, Kathleen, op. cit., pp. 570 y 571. 
se dio por ganador de las elecciones presidenciales a Thomas Dewey por sobre Harry Truman, poniendo de relieve graves errores de diseño metodológico y obligando a reorganizar y a replantear los procedimientos de indagación que estaban siendo utilizados..$^{24}$ La respuesta vino dada en la forma de la creación de los primeros códigos de ética y en el perfeccionamiento de la metodología de investigación cuantitativa. La AAPOR prontamente adquirirá un rol fundamental en esta materia. ${ }^{25} \mathrm{El}$ resultado será tal que ya a partir de 1952, el porcentaje de error promedio de las encuestas Gallup fluctuará comúnmente alrededor del $3 \%,{ }^{26} \sin$ perjuicio de que los errores que ha habido han sido muy importantes, como la falsa victoria de Bush en el voto popular del año 2000 o más recientemente en 2016, con la falla de los sondeos para predecir el fenómeno Trump. ${ }^{27}$ Cada vez que se presentan casos como éstos, reviven las críticas que piden legislar acerca del tema, pero la fuerza normativa que en el constitucionalismo norteamericano posee la Primera Enmienda, que protege la libertad de expresión, supone un freno infranqueable para cualquier intento de restringir la libertad de prensa. ${ }^{28}$ Como resultado, la libertad frente al Estado de la que goza la industria de opinión en Estados Unidos es casi total. Esta libertad ha sido defendida en más de una oportunidad por distintos tribunales federales, los cuales, con ocasión de algunos intentos aislados de restringir las exit polls a nivel estatal, una y otra vez han invariablemente reafirmado desde los años ochenta la inconstitucionalidad de estas barreras. ${ }^{29}$

24 Frankovic, Kathleen, "Opinion Polls and the Media in the United States", en Holtz-Bacha, Christina y Strömbäck, Jesper (eds.), Opinion Polls and the Media, Reflecting and Shaping Public Opinion, Nueva York, Palgrave Macmillan, 2012, pp. 115 y ss.

25 La WAPOR, por su parte, nacida de la misma conferencia junto a la AAPOR, se encargará de la exportación de estos principios y estándares de trabajo hacia el resto del mundo, a la vez que servirá de punto de encuentro para los encuestadores de otras naciones. Véase Baxter, Richard, "The Culture of AAPOR", en Sheatsley, Paul B. y Mitofsky, Warren J. (eds.), A Meeting Place, The History of the American Association for Public Opinion Research, Oakbrook Terrace, Illinois, AAPOR, 1992, p. 200. Lo anterior explica en parte tanto la influencia transfronteriza que han tenido las características del mercado estadounidense como la marcada postura antirregulatoria que frecuentemente manifiesta la WAPOR.

26 Streb, Matthew J. y Genovese, Michael A., op. cit., p. 6.

27 AAPOR, An Evaluation of 2016 Election Polls in the U.S., Oakbrook Terrace, Illinois, 2017, pp. 19 y ss., disponible en: https://www.aapor.org/Education-Resources/Reports.aspx, consultado el 27 de noviembre de 2018.

28 Donsbach, Wolfgang y Hartung, Uwe, op. cit., p. 434.

29 Gupta, Rani, "Questions of Constitutionality", Reporters Committee for Freedom of the Press, The News Media \& The Law, Washigton D. C., edición de invierno de 2007, p. 42, dis- 


\section{Canadá}

A diferencia de lo que ocurre en Estados Unidos de América, en Canadá la discusión respecto a la regulación de las encuestas se ha producido en torno a la protección de una comprensión robusta del derecho al sufragio. ${ }^{30}$ Así, al igual que lo que ocurre con la prohibición de vender alcohol durante la época electoral, se suele entender que la previsión de un "periodo de reflexión" antes de las elecciones y hasta el cierre de las urnas tiene por objetivo asegurar el ejercicio del voto en condiciones de racionalidad. Desde 1936 hasta 1993 había existido en este país un blackout de un día previo a los comicios aplicable sólo a los canales de televisión. ${ }^{31}$ En 1993, la aprobación de la Canadian Elections Act, una reforma de envergadura mayor al sistema de elecciones, aumentó este plazo a tres días e hizo extensible la prohibición a toda la población. A partir de entonces, el blackout canadiense ha oscilado entre los dos y tres días, hasta llegar a 2016, en que fue reducido a sólo un día. En forma adicional, la ley exige la publicación de la ficha técnica, que incluye la identificación del responsable de la encuesta y prohíbe su publicación en el extranjero, estableciendo multas para quien contravenga esta disposición. ${ }^{32}$ Con todo, la constitucionalidad de estas medidas ha sido desafiada en varias ocasiones. ${ }^{33}$ Fue, por ejemplo, en el caso Thomson Newspapers vs. Canada (1998) que la Corte Suprema Canadiense falló en contra del blackout original de tres días obligando a su reducción, puesto que su extensión excedía con creces el periodo de reflexión necesario y que por lo tanto éste constituía una violación injustificada de la libertad de expresión. Los potenciales beneficios intangibles no eran suficientes para justificar los daños tangibles de la prohibición. Por otra parte, se señaló que la consideración del votante más "desinformado y despistado" no podía servir para sentar el estándar nacional a la hora de

ponible en: https://www.rcfp.org/journals/the-news-media-and-the-law-winter-2007/questionsconstitutionality/, consultado el 20 de marzo de 2019.

30 Feasby, Colin C. J., op. cit., p. 243.

31 Trudel, Pierre y Abran, France, "The Legal and Constitutional Framework for Regulating Election Campaign Broadcasting", en Fletcher, Fred (ed.), Election Broadcasting in Canada, Toronto, Dundurn Press, 1991, vol. 21, pp. 118 y 119.

32 Canadian Elections Act, Clause 330.

33 Feasby, Colin C. J., op. cit., pp. 259 y ss. 
decidir sobre esta materia. ${ }^{34}$ Por último, este tribunal dictaminó en 2006 que la prohibición también era aplicable a los blogs en Internet. ${ }^{35}$

\section{España}

La Ley Orgánica 5/1985 del Régimen Electoral General establece en su artículo 69 el régimen de publicación de encuestas electorales, aplicable sólo durante el periodo de campaña electoral y compuesto por una serie de disposiciones tendentes a evitar la adulteración de los resultados que éstas arrojen. ${ }^{36}$ Con este fin, se exige la publicación de la ficha técnica, la identificación del encuestador y el texto íntegro de las preguntas planteadas. La norma otorga a la Junta Electoral Central amplias facultades de fiscalización en esta materia, pudiendo incluso, si lo juzgase conveniente, requerir del organismo encuestador información técnica complementaria con respecto a la encuesta realizada. El medio que difundiera sondeos con infracción a estos requisitos estará obligado a publicar las rectificaciones correspondientes. Asimismo, la norma prohíbe la publicación, difusión y reproducción de encuestas por cualquier medio de comunicación durante los cinco días anteriores al de la votación. Una particularidad de la ley española tiene relación con la gravedad de las sanciones que se prevén: las infracciones a esta normativa son constitutivas de delito y se castigan con penas de prisión que van de tres meses a un año, además de la imposición de multas administrativas. En general, puede decirse que la doctrina ha respaldado estas normas con argumentos basados en el rol público que juegan las encuestas y la consecuente necesidad de que éstas sean capaces de entregar datos confiables. ${ }^{37}$ Sin embargo, en los últimos años un sector relevante de la doctrina, más en línea con los estándares desarrollados por ESOMAR o WAPOR, ha sido enfático en denunciar la inutilidad de recu-

34 Thomson Newspapers Co. vs. Canada (Attorney General) (1998), 1 S.C.R. 877, disponible en: https://scc-csc.lexum.com/scc-csc/scc-csc/en/item/1621/index.do, consultado el 20 de marzo de 2019.

35 ACE Electoral Knowledge Network, Elecciones y medios de comunicación, 2018, disponible en: http://aceproject.org/ace-es/topics/me/meb/meb05/meb05c, consultado el 27 de noviembre de 2018.

36 López, Miguel Ángel et al., op. cit., pp. 234 y ss.

37 Gálvez Muñoz, Luis A., "Organismos de sondeos, encuestas electorales y derecho", Revista de Estudios Políticos, Madrid, 110, 2000, pp. 120 y ss.

Esta obra está bajo una Licencia Creative Commons

Atribución-NoComercial-SinDerivar 4.0 Internacional, IIJ-UNAM. 
rrir a la técnica del blackout frente a la masificación del acceso a Internet. ${ }^{38}$ Así, en abril de 2018, el Tribunal Supremo español, con ocasión del reclamo de la imposición de una multa tuvo la oportunidad de pronunciarse sobre el embargo de cinco días en orden a precisar que también caben dentro de esta prohibición aquellos estudios que no reúnan los requisitos legales para ser considerados encuestas, pero además y por sobre todo, señaló que

...la Junta Electoral Central es consciente de que, quizás, fuese pertinente suprimir la prohibición de publicar sondeos o encuestas en los días anteriores a los comicios, lo que implica en autos una apreciación de lege ferenda que explica que la sanción impuesta se haya fijado en su grado mínimo. ${ }^{39}$

\section{Francia}

El caso francés constituye tal vez una de las legislaciones más completas y estrictas en la materia, siendo comúnmente citado en la literatura especializada por este motivo. Conocidos en la industria son los amplios poderes que la Ley 77-808 (1977) ha entregado a la Comisión de Sondeos (Commission des Sondages), organismo de carácter administrativo e independiente encargado de velar por la veracidad, la transparencia y la rigurosidad técnica de las encuestas. ${ }^{40}$ Esta ley exige como requisito la publicación de la ficha técnica, que incluye una declaración que dé cuenta de la falibilidad de los sondeos y del margen de error. Antes de poder difundir la encuesta, sin embargo, y éste es el elemento distintivo de la regulación francesa, debe ser depositada en la Comisión, indicando el objetivo de ésta con información adicional a la ficha técnica, la cual podrá ser consultada

38 Sierra Rodríguez, Javier, "Regulación electoral de los sondeos a pie de urna en España: asignaturas pendientes y obsolescencia ante las nuevas formas de comunicación", Comunitania. Revista Internacional de Trabajo Social y Ciencias Sociales, Madrid, núm. 7, enero de 2014, pp. 105 y ss. Asimismo, González, María Holgado, "Publicidad e información sobre elecciones en los medios de comunicación durante la campaña electoral", Teoría y Realidad Constitucional, Madrid, núm. 40, 2017, pp. 480-483.

39 Sentencia Administrativa núm. 626/2018, Tribunal Supremo de España, Sala de lo Contencioso, Sección 4, Rec. 3564/2016 del 18 de abril de 2018. Considerandos décimo tercero y siguientes.

40 Loi no. 77-808 du 19 juillet 1977 relative à la publication et à la diffusion de certains sondages d'opinion. 
por cualquier persona, pues la Comisión publica esta información en su página institucional. La encuesta que no cumpla con estos requisitos podrá en cualquier momento ser rectificada por la Comisión y se notificará esta circunstancia a las agencias de prensa, las que deberán publicar una actualización del sondeo a la brevedad, procurando llegar a una audiencia equivalente a la que tuvo la publicación original. En forma adicional, un decreto del Consejo de Estado fijó en 1980 una serie de normas aplicables directamente al diseño metodológico de estos estudios, el cual impidió entre otros recursos el uso de preguntas capciosas. ${ }^{41}$ Hasta 2002 regía el embargo de una semana aplicable a la publicación, distribución y comentario de las encuestas, reducido ese año a dos días, cambio que si bien contó en un principio con la aprobación del público general y de la doctrina ${ }^{42}$ pronto se vio obstaculizado gracias a la irrupción de Internet. En efecto, a partir de 2007 y a pesar de que la contravención a esta ley implica una multa de 75,000 euros, se hizo cada vez más difícil para las autoridades controlar la difusión de las encuestas en medios extranjeros y en redes sociales como twitter. ${ }^{43}$ En consecuencia, una reforma legal llevada a cabo en 2016 restringió la aplicación de la ley al territorio nacional.

\section{Colombia}

En 1985 Colombia legisla por primera vez acerca de las encuestas electorales, instituyendo desde el comienzo una regulación bastante estricta. En efecto, además de exigir la publicación de la encuesta en su totalidad con indicación de la persona u organismo responsable, debía publicarse la ficha técnica completa, incluyendo la fuente de financiamiento y el margen de error. Se impuso, por otra parte, un periodo de embargo no sólo a la publicación, sino también a la elaboración de encuestas en los 30 días anteriores a una elección, limitación extensa considerando la media a nivel mundial, pero no tan inusual en comparación a la media latinoamericana, donde la mayoría de los países han establecido embargos de siete días o más. ${ }^{44}$ Pese a esto último, en 1993 la Corte Constitucional Colombiana de-

\footnotetext{
41 López, Miguel Ángel et al., op. cit., p. 234.

42 Gerstlé, Jacques, La comunicación política, trad. de Hernán Soto, Santiago de Chile, LOM Ediciones, 2005, pp. 64 y 65.

43 Sierra Rodríguez, Javier, op. cit., p. 104.

44 Esomar \& Wapor, op. cit., p. 17. 
claró inconstitucional la duración del embargo por considerarlo excesivo $\mathrm{y}$, por ende, atentatorio contra la libertad de expresión. ${ }^{45}$ La reacción del Congreso fue la publicación de la Ley 130 (marzo de 1994), cuyo artículo 30 redujo el número de días de embargo a uno. Esta nueva Ley mantuvo las obligaciones de publicar información y dio origen al Consejo Nacional Electoral, organismo con facultades normativas y de fiscalización, quien estableció la obligación de inscripción en el Registro Nacional de Encuestadores y aumentó la injerencia estatal en el diseño de las encuestas. Ello ha recibido constantes cuestionamientos por parte de la comunidad de encuestadores, por tratarse, según ellos, de una forma de censura previa. ${ }^{46}$ En 2005, el embargo subió a siete días pero sólo en elecciones en que el presidente en ejercicio fuera también candidato ${ }^{47}$ cambio tenido a bien por la Corte Constitucional en aplicación de criterios de proporcionalidad en la restricción. ${ }^{48}$ Por otro lado, desde 2015 duerme en el Senado el resistido Proyecto de Ley 114, el cual, entre otras cosas, busca endurecer todas estas medidas, aumentando a 10 días la duración del embargo para cualquier elección popular. ${ }^{49}$

\section{Chile}

En Chile el desarrollo de la industria de la opinión pública creció y se profesionalizó a partir de los años cincuenta ante el más absoluto silencio por parte del legislador. En 2016 Chile legisló al respecto, con la introducción de la Ley 20.900 (para el Fortalecimiento y Transparencia de la

\footnotetext{
45 Palomares García, Jorge, "El balanceo constitucional y la aplicación del método de proporcionalidad en las encuestas electorales", Revista de Derecho Público, Santiago de Chile, núm. 86, 2017, p. 91. Nótese que el reproche en este caso no fue dirigido al embargo propiamente tal, sino a su excesiva duración.

46 Restrepo, Javier, "Veda a las encuestas electorales: una mirada a la situación y la opinión sobre el tema en Colombia y algunas comparaciones a nivel global", Ipsos Public Affairs, Ipsos-Napoleón Franco, Bogotá, 2012, p. 6.

47 Ley 996 de 2005 (Ley de Garantías Electorales).

48 Palomares García, Jorge, op. cit., pp.95 y ss.

49 El Nuevo Siglo, Regulación de encuestas genera polémica política, Bogotá, 11 de junio de 2016, disponible en: https://www.elnuevosiglo.com.co/articulos/6-2016-regulaci\%C3\%B3nde-encuestas-genera-pol\%C3\%A9mica-pol\%C3\%ADtica, consultado el 29 de noviembre de 2018; Palomares García, Jorge, op. cit., p. 84.
} 
Democracia), el cual estableció un embargo de 15 días. ${ }^{50}$ Se designó como responsables en caso de contravención al blackout tanto al director del órgano de prensa, radioemisora o canal de televisión que publicare las encuestas como a la empresa propietaria o concesionaria del respectivo medio. La sanción que se fijó corresponde a una multa con un tope de 15,000 USD, cuya aplicación fue entregada al Servicio Electoral (Servel). ${ }^{51}$ Esta prohibición ha sido duramente criticada por parte de todos los sectores del mundo político y encuestador. Incluso el Poder Ejecutivo quiso distanciarse de la versión final de esta Ley, pues en su redacción original, el embargo sugerido era de tan solo cuatro días. El referido aumento a 15 días ocurrió en la tramitación parlamentaria del proyecto de ley, ${ }^{52}$ y fue aprobada sin discusión y por unanimidad. ${ }^{53} \mathrm{El}$ Tribunal Constitucional, al conocer de la constitucionalidad del embargo, a pesar de calificarlo como una restricción que afecta a las libertades de información y de expresión, consideró que dicha restricción era perfectamente lícita, pues el texto constitucional no consagra una protección absoluta de estos derechos. Antes bien, las restricciones podrán ser impuestas en la medida en que se funden en una "finalidad constitucional legítima". ${ }^{54}$ En el caso del embargo, esta finalidad consistiría, en primer lugar, en la consecución de una igualdad de oportunidades en materia electoral; $y$, en segundo lugar, la evitación de la manipulación de los resultados y los electores indecisos. Adicionalmente, la libertad de expresión, sostuvo el Tribunal, se vería afectada en esta materia de un modo meramente colateral. ${ }^{55} \mathrm{El}$ Tribunal observó que la regulación legal no contenía reglas básicas que versaran sobre la seriedad y la forma de producción de las encuestas electorales. ${ }^{56}$ Pese a la labor justificatoria

\footnotetext{
50 Ley 20.900, de 2016.

51 Ley 20.938, de 2016, artículo único.

52 Comisión Especial Investigadora del Senado, "Historia de la Ley núm. 20.900", Segundo Informe de Comisión Especial, 4 de diciembre de 2015, Informe de la Comisión Especial en sesión 90, Legislatura 363, Indicación núm. 70, pp. 4, 38 y 39.

53 Ibidem, 12 de enero de 2016, Discusión en Sala, Diario de Sesión en Sesión 91, Legislatura 363 , pp. 17,18 y $34-39$.

54 Sentencia del Tribunal Constitucional de Chile Rol núm. 2.981, del 31 de marzo de 2016. Considerandos sexagésimo tercero y ss.

55 Idem.

56 Ibidem, Considerandos sexagésimo octavo y sexagésimo noveno.
}

Esta obra está bajo una Licencia Creative Commons

Atribución-NoComercial-SinDerivar 4.0 Internacional, IJJ-UNAM. 
del TC, una vez puesta en conocimiento de la opinión pública, esta nueva normativa se vio enfrentada a una amplia resistencia. ${ }^{57}$

\section{LA REGULACIÓN DESDE UNA PERSPECTIVA TEÓRICA: EL ARGUMENTO DELIBERATIVO}

\section{La crítica a la regulación}

Tal como ya se adelantara, los instrumentos legales que regulan las encuestas electorales en el derecho comparado han sido objeto de críticas. La más recurrente es que ellos constituirían una afectación indebida a un conjunto de libertades básicas, garantizadas por los más diversos textos constitucionales y por tratados internacionales. En primer lugar, estos instrumentos restringirían la libertad de la empresa o institución, que elabora una encuesta electoral de divulgar los resultados de su investigación. Asimismo, afectarían la libertad de los medios de comunicación social de publicar esa información, restringiendo así, no sólo la libertad de expresión y de prensa sino que también el derecho de la ciudadanía a informarse sobre el proceso electoral en curso. ${ }^{58}$ En particular, el blackout es visto como un instrumento especialmente invasivo, puesto que impide de manera absoluta la publicación de encuestas electorales dentro de los plazos establecidos en las respectivas leyes y sería, según sus críticos, una forma de censura previa, inaceptable en un Estado democrático. Quienes critican las restricciones a las encuestas electorales no solamente lo hacen con base en la cantidad, sino tambén en la intensidad de las libertades afectadas. Además, tal como adelantamos, señalan que no existe evidencia empírica que demuestre a satisfacción los peligros asociados a las encuestas que justifiquen sus restricciones. ${ }^{59}$ Por todas estas razones, sostienen

57 Cooperativa, Encuestadores rechazan normativa que prohíbe publicar mediciones 15 dias antes de elecciones, Santiago de Chile, Cooperativa.cl. 29 de junio de 2017, disponible en: http://www.cooperativa.cl/noticias/pais/politica/encuestas/encuestadores-rechazan-nor mativa-que-prohibe-publicar-mediciones-15-dias/2017-06-29/064757.html, consultado el 26 de febrero de 2018).

58 Donsbach, Wolfgang y Hartung, Uwe, op. cit., pp. 433 y 434.

59 Tales peligros incluirían el efecto horse race, que consiste en que los electores indecisos tienden a sumarse a quienes aparecen liderando las encuestas electorales, distorsionando el resultado de las elecciones. Además, reforzarían la sensación de inevitabilidad de los resulta- 
que una regulación de las encuestas electorales en términos de blackout no pasaría ni el más generoso de los test de proporcionalidad. Mucho más aceptable en términos del costo para las libertades comunicativas es la regulación en términos de disclosure. No es raro, en este sentido, identificar autores que razonadamente rechazan el blackout pero exigen el disclosure en términos de fortalecimiento de las condiciones democráticas de la opinión pública con un costo relativamente menor para el ejercicio de las libertades comunicativas involucradas. ${ }^{60}$

Como se ha visto, la crítica a la regulación de las encuestas ha sido presentada fundamentalmente en términos de afectación de derechos fundamentales. Esa forma de presentar el problema ha llamado a un análisis de la constitucionalidad de dicha regulación en cuanto a la afectación de dichos derechos, típicamente en clave de proporcionalidad, prestando atención a la racionalidad, necesidad, intensidad y duración de las mismas. ${ }^{61}$ La regulación de las encuestas electorales ha sido objeto de preocupación de los órganos de protección internacional de los derechos humanos más o menos en los mismos términos. ${ }^{62}$ En estos casos, la perspectiva adoptada ha estado principalmente centrada en el grado y las modalidades de afectación de la libertad de expresión e información.

El argumento desarrollado en esta sección quiere adoptar una aproximación diferente. Haciendo eco de las propuestas de lo que ha sido denominado constitucionalismo deliberativo, ${ }^{63}$ creemos que la reconstrucción del problema centrada en la afectación de derechos fundamentales involucrados privilegia una comprensión liberal por sobre una comprensión

dos; promoverían el voto estratégico; reducirían la deliberación y aumentarían la posibilidad de utilizar las encuestas para manipular las decisiones del electorado. Véase Orr, Graeme y Levy, Ron, op. cit., pp. 321-326.

60 Por ejemplo, Corzo Sosa, Edgar, op. cit.; Hernández Valdez, Alfonso, "Efectos, errores y regulación de las encuestas preelectorales: una política de transparencia para el caso mexicano", Confines de Relaciones Internacionales y Ciencia Política, México, vol. 9, núm. 18 juniodiciembre de 2013, pp. 59-85.

61 Véase, como ejemplo de este tipo de análisis, Palomares García, Jorge, op. cit.

62 Véase una robusta síntesis, aunque no del todo actualizada, en Organización de Estados Americanos, "Libertad de expresión y procesos electorales: el caso de las encuestas de opinión y los sondeos de boca de urna", Informe anual de la Comisión Interamericana de Derechos Humanos 2005, Washington D. C., Informe de la Relatoría para la Libertad de Expresión, 2005, vol. II.

63 Véase, por ejemplo, Kong, Hoi y Levy, Ron, "Deliberative Constitutionalism", The Oxford Handbook of Deliberative Democracy, Cambridge, Cambridge University Press, 2017. 
democrática del valor de las libertades comunicativas. El argumento que sigue tiene justamente la finalidad de atacar esa forma de presentar el problema, y plantear una alternativa diferente de comprender la relación entre las libertades comunicativas y la garantía de una democracia deliberativa robusta. Busca pensar en ellas, no como dos fuerzas en conflicto sino como dos aspectos de una misma práctica constitucional. Ello no niega que a la hora de solucionar conflictos de constitucionalidad un aparato de análisis basado en el conflicto entre derechos y objetivos gubernamentales sea adecuado, sino que busca reivindicar la idea que los límites de los derechos deben ser examinados con atención al fundamento que estos derechos buscan instanciar, en este caso el de la libertad política, lo que puede llevar a concluir que los derechos sirven mejor a dicho interés cuando adoptan un carácter limitado.

\section{El argumento deliberativo}

La respuesta convencional que se ha dado a la crítica de la regulación de las encuestas electorales, a nuestro juicio correcta, descansa en el carácter esencialmente deliberativo de la democracia. El ideal democrático, esto es, que sea el pueblo el que decida por sí mismo aquellos asuntos relevantes para la vida en común, requiere que los ciudadanos tomen decisiones políticas sobre la base de una discusión pública en la que se enfrente el conjunto de opiniones, ideas y visiones existentes en la sociedad. Desde este punto de vista, la libertad de expresión e información tiene una función política fundamental. Su propósito es contribuir a la formación de una ciudadanía informada, que sea capaz de comprender el contenido y la relevancia de los asuntos que se discuten en la esfera pública, de distinguir las posiciones que allí se enfrentan y las consecuencias que de ellas se siguen para la vida en común. Vista así, la libertad de expresión e información, antes que resguardar un interés subjetivo por expresar ideas u opiniones sin restricciones en la esfera pública, resguarda un interés colectivo: ${ }^{64} \mathrm{el}$ interés en que todo asunto de relevancia política se someta a una discusión a) informada, b) balanceada, y c) comprehensiva. Mientras a) requiere que los argumentos que se invoquen para defender una u otra posición es-

64 Para el desarrollo de un argumento temprano de este punto desde la teoría democrática de la libertad de expresión, véase Meiklejohn, Alexander, Political Freedom: The Constitutional Powers of the People, Nueva York, Oxford University Press, 1965, p. 26. 
tén apoyados en evidencia razonable, $b$ ) requiere que todo argumento sea debidamente contrastado con argumentos que se le opongan, y $c$ ) requiere que todas las posiciones defendidas por segmentos relevantes de la población reciban la debida exposición. ${ }^{65}$

Llevado al terreno de los procesos electorales, el argumento deliberativo busca asegurar la creación de un ambiente propicio para que la ciudadanía se forme una opinión informada, sobre las posiciones de los candidatos sobre el conjunto de temas que se levantan durante las elecciones. Las encuestas electorales son vistas como un obstáculo para lograr lo anterior. El problema no consiste necesariamente en sus (potenciales) efectos microelectorales mencionados más arriba. Desde una perspectiva deliberativa, el problema es el tipo de deliberación y de discusión que las encuestas propician. De acuerdo con Susan Herbst, las encuestas hacen que la discusión política se torne superflua, ya que, crean la ilusión de que la ciudadanía se ha pronunciado de un modo definitivo y categórico respecto de una cuestión determinada. ${ }^{66}$ Los resultados arrojados por las encuestas, en otras palabras, son tratados como hechos sobre un aspecto determinado de la realidad política. Estos hechos no sólo condicionan las estrategias políticas de los candidatos, sino que también delimitan el ámbito y el tipo de discusión que se da durante periodos electorales. De acuerdo a Orr y Levy, en un sistema dominado por encuestas electorales la política se reduce a una teoría de juegos donde el enfrentamiento político se define por la posición relativa, que los candidatos ocupan en las encuestas, y en el que la reflexión electoral se circunscribe fundamentalmente al análisis sobre las estrategias que han llevado a unos y a otros candidatos a ocupar tales posiciones ${ }^{67}$ Así, la discusión sobre asuntos sustantivos, aquella que permite que los ciudadanos tomen decisiones reflexivas construidas sobre la base de una discusión informada, balanceada y comprehensiva, como lo demanda la deliberación democrática, queda relegada a un segundo plano. $\mathrm{Al}$ girar en torno al vaivén y ritmo de las encuestas, un escenario electoral dominado por ellas produce la constricción de la esfera pública. Tal constricción disminuye las posibilidades de que los agentes políticos, en este

65 Fishkin, James S. y Luskin, Robert C., "Experimenting with a Democratic Ideal: Deliberative Polling and Public Opinion”, Acta Politica, Londres, 40 (3), 2005, p. 249.

66 Herbst, Susan, Numbered Voices: How Opinion Polling Has Shaped American Politics, Chicago, University of Chicago Press, 1993, p. 166.

67 Orr, Graeme y Levy, Ron, op. cit., p. 335. 
caso los ciudadanos, tomen decisiones sobre la base de su propia reflexión a partir de un debate enfocado en los asuntos que definen nuestra vida en común. ${ }^{68}$

\section{3. ¿Está la publicación de encuestas realmente amparada en la libertad de expresión?}

En este contexto, la pregunta sobre si el Estado ha o no de regular las encuestas electorales puede circunscribirse, desde la perspectiva de la teoría constitucional, a una discusión sobre el concepto de la libertad y el rol que le corresponde al Estado en la configuración de la esfera pública. Quienes rechazan tal regulación porque afecta la libertad (la de las empresas encuestadoras, la de los medios de comunicación social y la de la ciudadanía), creen que la libertad consiste en la ausencia de interferencia externa en la esfera de decisiones y acciones de un agente individualmente considerado. Bajo este concepto liberal (negativo) de la libertad, ella se ve restringida, siguiendo a Isaiah Berlin, cada vez que la acción de un agente impide a otro hacer algo que de otro modo podría haber hecho. ${ }^{69}$ Así, las leyes que regulan las encuestas electorales interfieren en la libertad de las instituciones dedicadas al mercado de las encuestas y en los medios de comunicación social. A su vez, interfieren en la libertad de los ciudadanos puesto que les impide recibir información que de otro modo podrían haber recibido. En sintonía con la doctrina del libre mercado de las ideas, que rechaza toda forma de regulación de las expresiones que se vierten en la esfera pública como una amenaza al desarrollo del conocimiento y la búsqueda de la verdad, el concepto de libertad como no interferencia es extremadamente sensible a cualquier tipo de regulación legal de la expresión. ${ }^{70}$ Por lo mismo, estas leyes deben ser, de acuerdo con sus críticos, debidamente ponderadas para identificar si sus restricciones son propor-

68 Véase una reflexión más general sobre la necesidad de hacer congeniar a la libertad de expresión con la deliberación, en Muñoz, Tania Celina Vásquez, "Libertad de expresión y comunicación electoral: vías para un modelo deliberativo", Revista Justicia Electoral, México, núm. 15, 2015, pp. 245-277.

69 Berlin, Isaiah, "Two Concepts of Liberty”, en Hardy, Henry (ed.), Liberty, Oxford, Oxford University Press, 2002, p. 169.

70 Para un análisis sobre las consecuencias regulatorias de la doctrina del mercado de las ideas, véase Napoli, P. M., "The Marketplace of Ideas Metaphor in Communication Regulation”, Journal of Communication, Oxford, vol. 49, núm. 4, 1999, pp. 151-169. 
cionales a los fines que buscan alcanzar. En este juicio de ponderación se hace necesario encontrar evidencia empírica para demostrar los efectos negativos de las encuestas (bandwaggon, underdog, etcétera). Como tal evidencia no existe o es muy difícil de encontrar, un análisis de este tipo termina, en general, considerando que la regulación de las encuestas son una interferencia desproporcionada en la libertad. Esta posición es especialmente categórica en su rechazo al blackout como técnica de regulación de las encuestas electorales puesto que ella supone una restricción absoluta a la capacidad de las empresas encuestadoras de elaborarlas y/o difundirlas, así como de la ciudadanía de recibir la información en ellas contenidas. Tratándose del disclosure, esta posición puede ser más tolerante; sin embargo, para aceptar esta última técnica será necesario demostrar con claridad los efectos perjudiciales que las encuestas pueden generar en periodos electorales. Es por ello que antes que la regulación legal de las encuestas, esta posición privilegia mecanismos de autorregulación.

El problema de este concepto de libertad es que, si bien, no tolera la interferencia externa en la esfera de acción individual, en cambio sí tolera la existencia de estructuras de dominación. Fue, en efecto, el propio Thomas Hobbes el que inventó este concepto de libertad para oponerlo a un concepto político de libertad (la libertad republicana) y para justificar su compatibilidad con la del poder absoluto del Leviathan. ${ }^{71}$ Esta compatibilidad se explica por el hecho de que la libertad apunta a un hecho esencialmente contingente: la ausencia de coacción o interferencia. Las estructuras de dominación no necesariamente generan interferencia y en cuanto no lo hagan, no constituyen afectaciones a la libertad. ${ }^{72}$ Como las encuestas electorales no fuerzan o impiden a un agente actuar o dejar de actuar de un modo determinado, ellas no constituyen un problema desde la perspectiva de la libertad como no-interferencia. ${ }^{73}$ Sin embargo, su falta de regulación

71 Para un análisis histórico sobre el concepto de libertad en Hobbes y el surgimiento del concepto liberal de libertad, véase Skinner, Quentin, Hobbes and Republican Liberty, Cambridge, Cambridge University Press, 2008.

72 Para un desarrollo sistemático y un contraste entre el concepto de libertad como no interferencia y como no dominación, véase Pettit, Philip, Republicanism: A Theory of Freedom and Government, Oxford, Oxford University Press, 1997, cap. 1.

73 Para un argumento que explora las relaciones de dominación que se siguen de la práctica de la libertad como no interferencia en los medios de comunicación social, véase Charney, John, The Illusion of the Free Press, Oxford, Hart Publishing, 2018, pp. 126-134. 
incrementa el riesgo de reforzar estructuras de dominación en el sistema político.

A diferencia del anterior, el argumento deliberativo puede construirse sobre un concepto de libertad como no-dominación. La dominación, a diferencia de la interferencia, no mira el hecho contingente y puntual de interferencia para calificar si hay o no una afectación a la libertad, sino que mira las estructuras en las que se enmarcan las relaciones humanas. Independiente de si existe o no interferencia, la libertad se ve afectada cuando esas estructuras permiten que un agente pueda interferir arbitrariamente en las decisiones de otro (aun cuando en la práctica no lo haga). Desde esta perspectiva las encuestas electorales son una afectación de la libertad puesto que aun si ellas no interfieren directamente en las decisiones de un agente, para que vote en uno u otro sentido, constriñen la discusión en la esfera pública y con ello el universo de posibilidades que permiten decidir en uno $u$ otro sentido. La no-dominación requiere de una esfera pública que promueva una discusión centrada en aquellos asuntos que son verdaderamente relevantes para tomar decisiones políticas. Requiere que la ciudadanía se enfoque en cuáles son las propuestas que unos y otros candidatos tienen sobre los asuntos que definen las bases de la convivencia política. Así, cuando el debate en periodos electorales se concentra en un juego de estrategias circunscrito a sus lógicas deportivas internas se afecta una libertad política fundamental: aquella que consiste en que la ciudadanía decida por sí misma, esto es, con base en motivos y razones fundadas que han sido debidamente articuladas y confrontadas en la esfera pública, y no en motivos puramente especulativos, como ha de conducirse el destino de la comunidad política durante un periodo determinado.

Desde la perspectiva de la libertad como no-dominación, el Estado tiene el deber de asegurar un ambiente propicio para el desarrollo de esta libertad política fundamental. La libertad de expresión e información - no entendida como un límite al ejercicio de la actividad estatal, que busca garantizar un derecho individual a manifestarse en la esfera pública, sino como una herramienta que habilita la capacidad del pueblo de decidir por sí mismo - es el medio que permite articular, a través de un debate informado, balanceado y comprehensivo las distintas visiones que se enfrentan en la arena política. Para lograr lo anterior el Estado debe, muchas veces, interferir en la estructura y organización de las prácticas comunicativas que se desarrollan en la esfera pública. Esta interferencia es perfectamente 
compatible con un sistema democrático y con la libertad (como no-dominación) y ampliamente utilizada para, entre otras cosas, evitar la concentración en la propiedad de los medios de comunicación social, estimular el pluralismo en el sistema informativo o para asegurar un debate informado durante periodos de elecciones a través de la regulación de la publicidad electoral y, por cierto, de las encuestas electorales. Evidentemente esta interferencia debe adoptar formas institucionalmente reconocidas como legítimas y en un sistema democrático es la ley la que cumple esta función legitimadora. Desde esta perspectiva la ley no es una afectación de la libertad, sino el medio a través del cual ella es asegurada.

\section{Una propuesta que garantiza el funcionamiento de la deliberación}

Bajo esta estructura conceptual podemos afirmar, en primer lugar, que la regulación legal de las encuestas electorales es, sin duda, una forma de interferencia en las decisiones y en las acciones de quienes se vinculan con la producción, difusión y recepción de encuestas electorales. Esta forma de interferencia es, sin embargo, un medio a través del cual se busca atacar la constricción de la esfera pública y fomentar una discusión informada, balanceada y comprehensiva, que gire en torno a asuntos sustantivos de la vida en común, en periodos electorales. Es una discusión de estas características la que permite que el pueblo decida con un mayor grado de libertad quiénes deben asumir el poder político. Es por ello por lo que desde un punto de vista deliberativo y sobre la base de un concepto de libertad como no-dominación, la regulación legal de las encuestas electorales no puede verse sólo como una afectación de libertades básicas, sino que también puede constituir una herramienta legítima para habilitar la libertad política de un pueblo que se expresa a través de las elecciones representativas. Debido a que este concepto de libertad no se concentra en la interferencia de los agentes que participan de los procesos electorales (activa y pasivamente), sino en desmantelar las estructuras de dominación, es tolerante a cualquier técnica de regulación que persiga tal propósito, y que no genere a su vez nuevas estructuras de dominación. Por este motivo, y a diferencia de la libertad como no interferencia, tolera indistintamente el blackout y el disclosure como técnicas constitucionalmente legítimas para reforzar la libertad política de la ciudadanía. 
Sobre la base de esta reflexión teórica, en torno a la importancia que la deliberación sobre asuntos sustantivos en periodos electorales tiene sobre la libertad política, puede realizarse una propuesta de racionalización de esta regulación que apunte tanto a su mejor fundamentación como a su vinculación racional con el sistema político y electoral. Esta propuesta parte de la base de que el derecho electoral nacional admite que el periodo de campaña electoral es un periodo de excepcionalidad política. Su excepcionalidad política permite que la protección de ciertos aspectos de la vida democrática de un país se agudice y otros aspectos se vean postergados de la primera línea de preocupación por parte del derecho. Uno de tales aspectos es, por supuesto, la libertad política. Por ejemplo, es sólo durante la campaña electoral que el Estado considera que es legítimo que los partidos y candidatos intenten convencer abiertamente, mediante propaganda electoral, a los ciudadanos de votar por ellos en la próxima elección. Por el contrario, fuera del periodo de campaña electoral, la legislación prohíbe tal propaganda. Esa prohibición es una interferencia razonable a la luz del interés en limitar la discusión propiamente electoral a un periodo restringido, donde los ciudadanos se ven expuestos masivamente a las propuestas de los candidatos en competencia. Es bajo la misma lógica, que parece razonable que durante los periodos de campaña electoral se creen instrumentos que tengan como propósito reforzar la discusión política y facilitar la toma de decisiones informadas. Tales formas de interferencia, como hemos visto, reducen los peligros de dominación de la esfera pública y con ello refuerzan una libertad que cobra especial importancia durante los periodos de elecciones. Tales instrumentos, como son en este caso la obligación de disclousure y el blackout, no deben ser, por tanto, entendidos como amenazas o restricciones a la libertad de expresión e información, sino como condiciones para su ejercicio en términos más armónicos con el correcto funcionamiento de una democracia deliberativa.

\section{CONCLUSIONES}

Cuando miramos al derecho comparado, podemos identificar junto a la existencia de una tradición que ha desestimado la necesidad de regulación legal de las encuestas electorales, al menos dos aspectos que han sido objeto de dicha regulación con la finalidad más o menos explícita de priorizar el proceso de deliberación preelectoral frente al derecho a la libertad de 
expresión e información que protegería la libertad de publicar encuestas electorales. En primer lugar, y buscando asegurar la calidad de la información provista, se ha exigido que los resultados de las encuestas sean acompañados por fichas técnicas o disclosures que provean información comprensible que permita juzgar, por parte de la ciudadanía, el valor informativo y científico de cada encuesta. Proveer información sobre la fecha en que se llevó a cabo la muestra, su tamaño, el margen de error de la metodología empleada, la formulación de las preguntas permite confiar en los resultados ofrecidos. En segundo lugar, se han fijado embargos o blackouts en los que la publicación de encuestas electorales se encuentra prohibida. Si bien este segundo tipo de limitación, cuando es aplicada solamente los días inmediatamente anteriores de la elección, no tiene el efecto deliberativo que podría tener su total prohibición durante el periodo de campaña, puede interpretarse como un indicio de que, contrariamente a la tesis del mercado de las ideas (o del mercado de información), que sostiene que la verdad emergerá de la libre competencia entre información vertida en el espacio público, la sustracción de información sí podría tener un efecto positivo tanto para la deliberación democrática como para la formación de un juicio político más profundo por parte de la ciudadanía sobre la crucial cuestión de quién llevará las riendas de la dirección política del Estado.

La deliberación, en especial aquella que se da en periodos de elecciones, es vital para el adecuado funcionamiento del sistema democrático. Ella permite que el cuerpo político pueda tomar decisiones informadas sobre la base de una discusión comprehensiva, balanceada y sustantiva. Esta es una libertad política fundamental que se ve afectada cuando la discusión que se da en la esfera pública es dominada por encuestas electorales que no han sido debidamente reguladas. En efecto, su falta de regulación no sólo podría aumentar el riesgo de que ellas sean manipuladas o tergiversadas con fines electorales, sino que además orienta el debate hacia cuestiones estratégicas y elitistas, alejándolo de consideraciones sustantivas sobre la vida en común. Desde esta perspectiva, las encuestas electorales constriñen la libertad política y por lo mismo su regulación no úncamente es compatible, sino que también es necesaria para asegurar tal libertad. 


\section{BIBLIOGRAFÍA}

Aalberg, Toril. y VAn Aelst, Peter, "Who is Afraid of Preelection Polls? How Perceptions of Polls Influence Support for Polling Regulations Among Elites", International Journal of Public Opinion Research, 26, núm. 4, 2014.

AAPOR, An Evaluation of 2016 Election Polls in the U.S., Oakbrook Terrace, 2017, disponible en: https://www.aapor.org/Education-Resources/ Reports.aspx, consultado el 27 de noviembre de 2018.

ACE Electoral KnOwledge Network, Elecciones y medios de comunicación, 2018, disponible en: http://aceproject.org/ace-es/topics/ me/meb/meb05/meb05c, consultado el 27 de noviembre de 2018.

AgUiAR, César, "Apuntes para una historia de los estudios de opinión pública en América Latina", en CORDERO, Rodrigo (ed.), La sociedad de la opinión, reflexiones sobre encuestas y cambio político en democracia, Santiago de Chile, Ediciones Universidad Diego Portales, 2009.

AlONSO, Belén, "Las encuestas en los medios. Un estudio sobre su regulación en Argentina desde el tecnicismo y la política", XXV International Congress Latin American Studies Association (LASA), Las Vegas, octubre de 2004.

ARRIAGADA, Eduardo, "Cómo se reportea una encuesta", Cuadernos Info, Pontificia Universidad Católica de Chile, núm. 8, 1993.

AstorgA, Luz María, "Lo que se esconde tras el mundo de las encuestas", Diario Concepción, 15 de enero de 2017, disponible en: https://www. diarioconcepcion.cl/sin-categoria/2017/01/15/lo-que-se-esconde-trasel-mundo-de-las-encuestas.html, consultado el 25 de febrero de 2018.

BAXTER, Richard, “The Culture of AAPOR”, en SHEATSLEY, Paul B. y MITOFSKY, Warren J. (eds.), A Meeting Place. The History of the American Association for Public Opinion Research, Oakbrook Terrace, Illinois, AAPOR, 1992.

BERLIN, Isaiah, “Two Concepts of Liberty”, en HARDY, Henry (ed.), Liberty, Oxford, Oxford University Press, 2002.

Biblioteca Nacional del Congreso de Chile, Encuestas electorales. Experiencia comparada, Santiago de Chile, Informe, 2012, disponible en: http://www.bcn.cl/obtienearchivo?id=repositorio/10221/16052/1/ 
Encuestas\%20electorales.\%20Experiencia\%20comparada_v5.doc, consultado el 26 de febrero de 2018.

Charney, John, The Illusion of the Free Press, Oxford, Hart Publishing, 2018.

Chung, Robert, The Freedom to Publish Opinion Poll Results. A Worldwide Update of 2012, Public Opinion Programme, The University of Hong Kong, World Association for Public Opinion Research, 2012.

COOPERATIVA, "Encuestadores rechazan normativa que prohíbe publicar mediciones 15 días antes de elecciones", Cooperativa.cl., Santiago de Chile, 29 de junio de 2017, disponible en: http://www.cooperativa.cl/ noticias/pais/politica/encuestas/encuestadores-rechazan-normativaque-prohibe-publicar-mediciones-15-dias/2017-06-29/064757.html, consultado el 26 de febrero de 2018.

CORDERO, Rodrigo y MARÍN, Cristóbal, "Los medios masivos y las transformaciones de la esfera pública en Chile", Documento de trabajo ICSO, núm. 7, año 2, agosto 2006.

CORDERO, Rodrigo y TAPIA, Gonzalo, "Sumando opiniones: antecedentes históricos y desarrollos metodológicos de la industria de la opinión pública en Chile", Documento de trabajo ICSO, núm. 15, año 3, abril de 2007.

Corzo SosA, Edgar, "Las encuestas electorales y los derechos fundamentales: Una primera reflexión", Cuestiones Constitucionales, México, núm. 16, 2007.

DAHLGAARD, Jens Olav et al., "How are Voters Influenced by Opinion Polls? The Effect of Polls on Voting Behavior and Party Sympathy", World Political Science, Copenhagen, vol. 12, núm. 2, 2016.

DONSBACH, Wolfgang y HARTUNG, Uwe, "The legal Status of Public Opinion Research in the World", en DONSBACH, Wolfgang y TRAUGOTT, Michael W. (eds.), The Sage Handbook of Public Opinion Research, Los Angeles, Sage Publications, 2008.

DONSBACH, Wolfgang, Who's Afraid of Election Polls? Normative and Empirical Arguments for the Freedom of Pre-election Surveys, Amsterdam, Foundation for Information, ESOMAR, 2001.

ESOMAR y WAPOR, Esomar/Wapor Guideline on Opinion Polls and Published Surveys, Lincoln, Nebraska, 2014, disponible en: https://www.esomar. org/uploads/public/knowledge-and-standards/codes-and-guidelines/ 
ESOMAR-WAPOR-Guideline-on-Opinion-Polls-and-Published-Surveys-August-2014.pdf, consultado el 26 de noviembre de 2018.

Esomar y Wapor, Freedom to Conduct Opinion Polls. A 2017 Worldwide Update, Lincoln-Nebraska, 2018, disponible en: https://wapor.org/publications/freedom-to-publish-opinion-polls/, consultado el 27 de noviembre de 2018.

Feasby, Colin C. J., "Public Opinion Poll Restrictions, Elections, and the Charter", U. Toronto Fac. L. Rev., Toronto, 55, 1997.

FISHKIN, James S. y LUSKIN, Robert C., "Experimenting with a Democratic Ideal: Deliberative Polling and Public Opinion", Acta politica, Londres, 40 (3), 2005.

FranKOVIC, Kathleen, "Exit Polls and Pre-Election Polls", en DONSBACH, Wolfgang y TRAUGOTT, Michael W. (eds.), The Sage Handbook of Public Opinion Research, Los Angeles, Sage Publications, 2008.

FRANKOVIC, Kathleen, "Opinion Polls and the Media in the United States", en Holtz-BACHA, Christina y STRÖMBÄCK, Jesper (eds.), Opinion Polls and the Media, Reflecting and Shaping Public Opinion, Nueva York, Palgrave Macmillan, 2012.

GÁlvEZ MuÑOZ, Luis A., "Las encuestas electorales y el debate sobre su influencia en las elecciones", Revista Mexicana de Opinión Pública, México, 11, 2011.

GÁlvEZ MuÑoz, Luis A., "Organismos de sondeos, encuestas electorales y derecho", Revista de Estudios Politicos, Madrid, 110, 2000.

GeRstLÉ, Jacques, La comunicación politica, trad. de Hernán Soto, Santiago de Chile, LOM Ediciones, 2005.

GonZÁLEZ, María Holgado, "Publicidad e información sobre elecciones en los medios de comunicación durante la campaña electoral", Teoría y Realidad Constitucional, Madrid, núm. 40, 2017.

GuPTA, Rani, "Questions of Constitutionality", Reporters Committee for Freedom of the Press, Washigton D. C., (de la edición de invierno de 2007 de The News Media \& The Law), disponible en: https://www. rcfp.org/journals/the-news-media-and-the-law-winter-2007/questionsconstitutionality/, consultado el 20 de marzo de 2019.

Herbst, Susan, Numbered Voices: How Opinion Polling Has Shaped American Politics, Chicago, University of Chicago Press, 1993. 
HERNÁNDEZ VALDEZ, Alfonso, "Efectos, errores y regulación de las encuestas preelectorales: una política de transparencia para el caso mexicano", Confines de Relaciones Internacionales y Ciencia Política, México, vol. 9, núm. 18, junio-diciembre de 2013.

COMISIÓN ESPECIAL InVESTIGADORA DEL SENADO, Historia de la Ley núm. 20.900, Segundo informe de la Comisión Especial, Senado, Discusión en Sala, Diario de Sesión en Sesión 91, Legislatura 363, 12 de enero de 2016, disponible en: https://www.bcn.cl/historiadelaley/nc/historia-dela-ley/4915/, consultado el 7 de mayo de 2018.

COMISIÓN ESPECIAL INVESTIGADORA DEL SENADO, Historia de la Ley núm. 20.900, Segundo Informe de Comisión Especial, Senado, Informe de Comisión Especial en Sesión 90, Legislatura 363, 4 de diciembre de 2015, disponible en: https://www.bcn.cl/historiadelaley/nc/historia-dela-ley/4915/, consultado el 7 de mayo de 2018.

HuneEus, Carlos, "Democracia versus Encuestocracia", Revista Mensaje, octubre de 2007.

Kong, Hoi, y Levy, Ron, "Deliberative Constitutionalism”, The Oxford Handbook of Deliberative Democracy, Cambridge, Cambridge University Press, 2017.

LÓPEZ, Miguel Ángel et al., "Sobre experiencia internacional en regulación y autorregulación de encuestas políticas y electorales de opinión pública. Lecciones para Chile", en FUENTE, Gloria de la (ed.), Calidad de la democracia, transparencia e instituciones politicas, Santiago de Chile, Segpres y Lom Ediciones, vol. 1.

MAYOL, Alberto y CRESPO, Francisco, "Estándares y regulaciones de las encuestas de opinión pública”, Revista Políticas Públicas, vol. 8, núm. $1,2015$.

MeIKlejohn, Alexander, Political Freedom: The Constitutional Powers of the People, Nueva York, Oxford University Press, 1965.

NAPOLI, P. M., "The Marketplace of Ideas Metaphor in Communication Regulation", Journal of Communication, Oxford, vol. 49, 4, 1999.

NAVIA, Patricio, "Public Opinion Polls in Chile", en GEer, John (ed.), Public Opinion and Polling Around the World: A Historical Encyclopedia, Nueva York, ABC-Clio, 2004.

ORGANIZACIÓN DE ESTADOS AMERICANOS, “Libertad de expresión y procesos electorales: el caso de las encuestas de opinión y los sondeos de 
boca de urna", Informe anual de la Comisión Interamericana de Derechos Humanos 2005, Washington D. C., Informe de la Relatoría para la Libertad de Expresión, 2005, vol. II.

ORR, Graeme Y LEVy, Ron, "Regulating Opinion Polling: A Deliberative Democratic Perspective”, UNSW Law Journal, Sydney, vol. 39, núm. 1, 2016.

PALOMARES GARCÍA, Jorge, "El balanceo constitucional y la aplicación del método de proporcionalidad en las encuestas electorales", Revista de Derecho Público, Santiago de Chile, núm. 86, 2017.

PeÑa MenA, Rodrigo de la, "El debate sobre las encuestas electorales en México en 2012”, Revista Mexicana de Opinión Pública, México, núm. 20, 2016.

PETERSEN, Thomas, "Regulation of Opinion Polls: AComparative Perspective", en Holtz-BACHA, Christina y StröMBÄCK, Jesper (eds.), Opinion Polls and the Media, Reflecting and Shaping Public Opinion, Nueva York, Palgrave Macmillan, 2012.

PetTIT, Philip, Republicanism: A Theory of Freedom and Government, Oxford, Oxford University Press, 1997.

"Regulación de encuestas genera polémica política", El Nuevo Siglo, Bogotá, 11 de junio de 2016, disponible en: https://www.elnuevosiglo. com.co/articulos/6-2016-regulaci\%C3\%B3n-de-encuestas-generapol\%C3\%A9mica-pol\%C3\%ADtica, consultado el 29 de noviembre de 2018.

RESTREPO, Javier, "Veda a las encuestas electorales: una mirada a la situación y la opinión sobre el tema en Colombia y algunas comparaciones a nivel global", Ipsos Public Affairs, Ipsos-Napoleón Franco, Bogotá, 2012.

SÁNCHEZ, Enrique Inti García, "Criterios legales y jurisdiccionales vinculados a encuestas electorales en México", Revista Mexicana de Estudios Electorales, México, núm. 16, 2016.

Servel, Acuerdo Consejo Directivo en respuesta a consulta núm. 14803, 22 de junio de 2017, disponible en: https://pbs.twimg.com/medial DDbKgwUXUAQamhF.jpg, consultado el 7 de mayo de 2018.

SIERRA RodríGUEZ, Javier, "Regulación electoral de los sondeos a pie de urna en España: asignaturas pendientes y obsolescencia ante las nue- 
vas formas de comunicación", Comunitania. Revista Internacional de Trabajo Social y Ciencias Sociales, Madrid, núm. 7, enero de 2014.

SKINNER, Quentin, Hobbes and Republican Liberty, Cambridge, Cambridge University Press, 2008.

Streb, Matthew J. y Genovese Michael A., "Polling and the Dilemmas of Democracy", en Streb, Matthew J. y Genovese, Michael A. (eds.), Polls and Politics, The Dilemmas of Democracy, Albany, State University of New York Press, 2004.

TRudel, Pierre y ABran, France, "The Legal and Constitutional Framework for Regulating Election Campaign Broadcasting", en FLETCHER, Fred (ed.), Election Broadcasting in Canada, Toronto, Dundurn Press, 1991.

VÁSQUEZ MuÑoz, Tania Celina, "Libertad de expresión y comunicación electoral: vías para un modelo deliberativo", Revista Justicia Electoral, México, núm. 15, 2015.

WAPOR, WAPOR, Statement on Chilean Proposed Ban on Polls, Lincoln, Nebraska, 28 de junio de 2017, disponible en: https://mailchi. mp/420d02917c40/wapor-statement-on-chilean-proposed-ban-onpolls? $=48$ ed52fb04, consultado el 29 de abril de 2018 .

Fecha de recepción: 28 de marzo de 2019.

Fecha de aceptación: 20 de mayo de 2019.

Esta obra está bajo una Licencia Creative Commons

Atribución-NoComercial-SinDerivar 4.0 Internacional, IIJ-UNAM. 